\title{
Infectious endocarditis caused by Nocardia sp.: histological morphology as a guide for the specific diagnosis
}

\author{
Authors \\ Jussara Bianchi Castelli ${ }^{1}$ \\ Rinaldo Focaccia Siciliano ${ }^{2}$ \\ Edson Abdala ${ }^{3}$ \\ Vera Demarchi Aiello ${ }^{1}$ \\ ${ }^{1} \mathrm{MD}, \mathrm{PhD}$, Laboratory of \\ Pathology, InCor, Medical \\ School, Universidade de São \\ Paulo (USP), Brazil \\ ${ }^{2} \mathrm{MD}$, Infection Control Unit, \\ InCor, Medical School, USP, \\ Brazil \\ ${ }^{3} \mathrm{MD}$, PhD, Liver Tranplant \\ Service, Gastroenterology \\ Department, Medical School, \\ USP, Brazil
}

Submitted on: 12/10/2010 Approved on: 04/9/2011

\footnotetext{
Correspondence to: Jussara Bianchi Castelli Laboratory of Pathotological Anatomy InCor - HCFMUSP Av. Dr. Eneas de Carvalho Aguiar, 44 05403-000, São Paulo, SP Brazil

jussara.castelli@incor.usp.br
}

We declare no conflict of interest.

(C2011 Elsevier Editora Ltda. All rights reserved.

\begin{abstract}
Nocardia is a rare opportunistic agent, which may affect immunocompromised individuals causing lung infections and exceptionally infective endocarditis (IE). There are few reports of IE caused by Nocardia sp., usually involving biological prostheses but rarely in natural valves. Its accurate microbiological identification may be hampered by the similarity with Rhodococcus equi and Corynebacterium spp. Here we report a case of native mitral valve IE caused by this agent in which the clinical absence of response to vancomycin and the suggestion of Nocardia sp. by histology pointed to the misdiagnosis of Corynebacterium spp. in blood cultures. The histological morphology can advise on the need for expansion of cultivation time and use of extra microbiological procedures that lead to the differential diagnosis with Corynebacterium spp. and other agents, which is essential to establish timely specific treatment, especially in immunocompromised patients.
\end{abstract}

Keywords: mitral valve; endocarditis, bacterial; Nocardia infections.

\section{INTRODUCTION}

Nocardia is a genus of the order Actinomycetales, which also belong to the genera Tsukamurella, Gordonia, Streptomyces, and Rhodococcus. Other closely related genera include Corynebacterium and Mycobacterium (bacilli). Tsukamurella spp. are often misidentified as Rhodococcus or Corynebacterium spp. ${ }^{1,2}$ The acid-fast resistance staining feature is present in the genera Nocardia (long filamentous bacteria with buds at right angles), Gordonia (coccus), Rhodococcus (coryneform bacillus) and Tsukamurella (bacillus short to long, unbranched), ${ }^{2}$ which alone can generate misdiagnosis if the morphological features are not considered.

Infection by Nocardia sp. usually manifests as acute suppurative, subacute or chronic. The main affected organs are the lungs, in the form of pneumonia with cavitation and drainage to the pleural space. Clinical manifestations also include tracheobronchitis, peritonitis, rectal abscess, sinusitis, mediastinitis, peritonitis, osteomyelitis, arthritis and rarely endocarditis, the latter usually associated with prior heart disease. The infection usually occurs in patients with prosthetic valve, and is very rare in native valves. Endocarditis by Nocardia sp. has an indolent course, low level of clinical suspicion, and the initial empiric antibiotic therapy is often not adequate. Valve replacement and culture of the vegetation are critical to prescribe a powerful and adequate antibiotic regimen. ${ }^{3}$

\section{CASE REPORT}

A 36 year-old male patient submitted to liver transplant for alcoholic cirrhosis one year and four months before, with five acute rejection episodes and using of immunosuppressive drugs (cyclosporine, azathioprine, hydrocortisone) for five months, presented with malaise and fever, undergoing investigation of an unknown focus of infection. He had received cefaclor for ten days with a slight improvement, with recrudescence of fever after completion. A transesophageal echocardiogram showed large sessile vegetation attached to the anterior leaflet of the mitral valve and had five blood cultures positive for Corynebacterium sp. allowing the diagnosis of infective endocarditis (IE). The patient was receiving ceftriaxone, which was replaced by vancomycin after blood culture results. He remained with fever and 
A

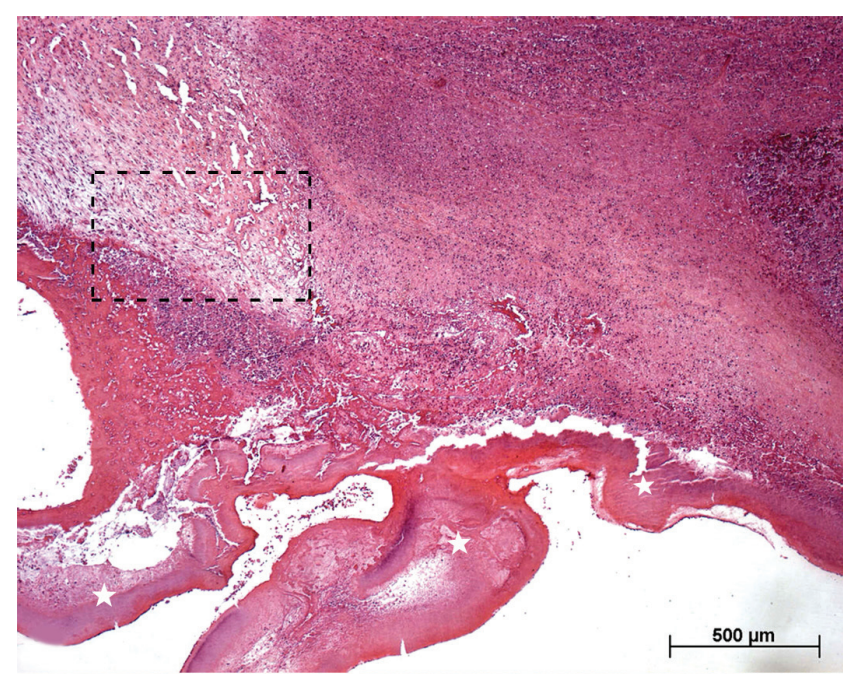

B

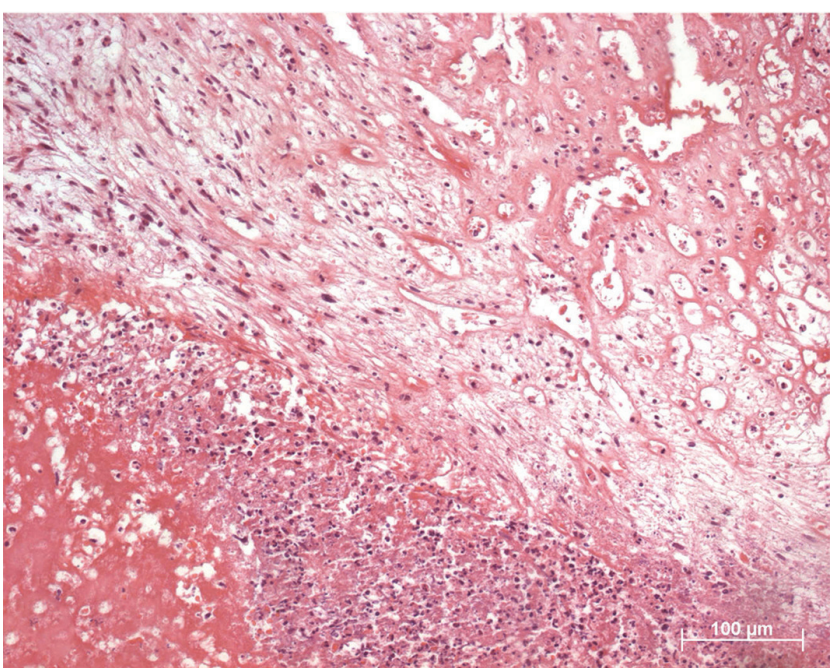

Figure 1: (A) Photomicrography of the mitral valve endocarditis presenting with large vegetation. Numerous filamentous bacteria entangled at the surface (white stars) are shown in detail in Figure 2. The region within the square is the base of the vegetation which is shown under higher magnification in Figure 1B. (hematoxylin-eosin, original magnification x5); (B) Higher magnification of the tissue shown in Figure 1A. The vegetation is composed of fibrin and neutrophils, but there is already granulation tissue at the base with neoformed vessels, indicating a process in chronification (hematoxylin-eosin, original magnification $\mathrm{x} 20$ ).

developed heart failure with progressive dyspnea even at rest. Emergency surgery was indicated due to IE with possible cordal rupture. The explanted mitral valve showed a vegetation measuring $1.5 \times 1.0 \times 0.8 \mathrm{~cm}$ on the atrial aspect of the anterior leaflet and rupture of some chordae tendinae (Figure 1). Histology confirmed IE with multiple Gram-positive and acid-fast-resistant filamentous bacteria, with buds at right angles, consistent with Nocardia sp. (Figure 2). The patient received trimethoprim-sulfamethoxazole and underwent new valve

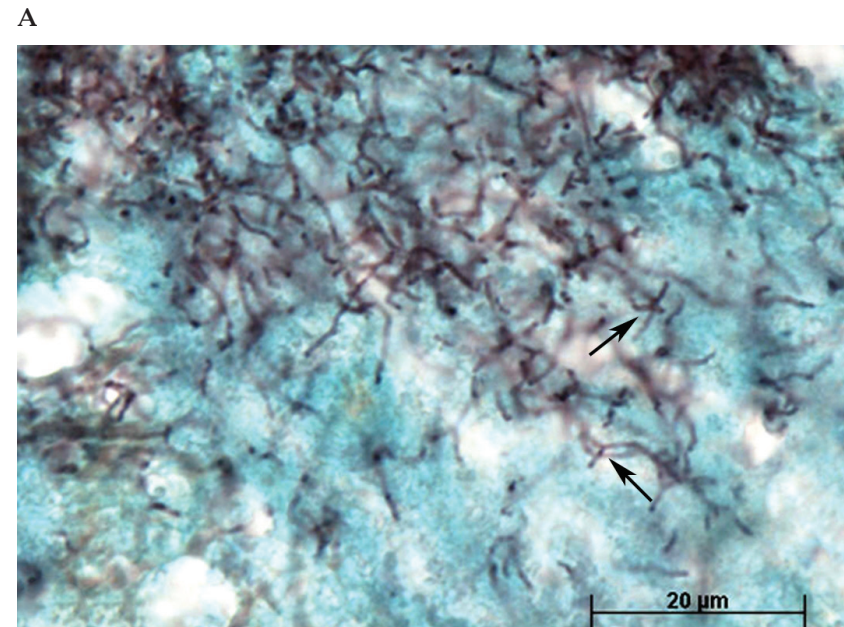

B

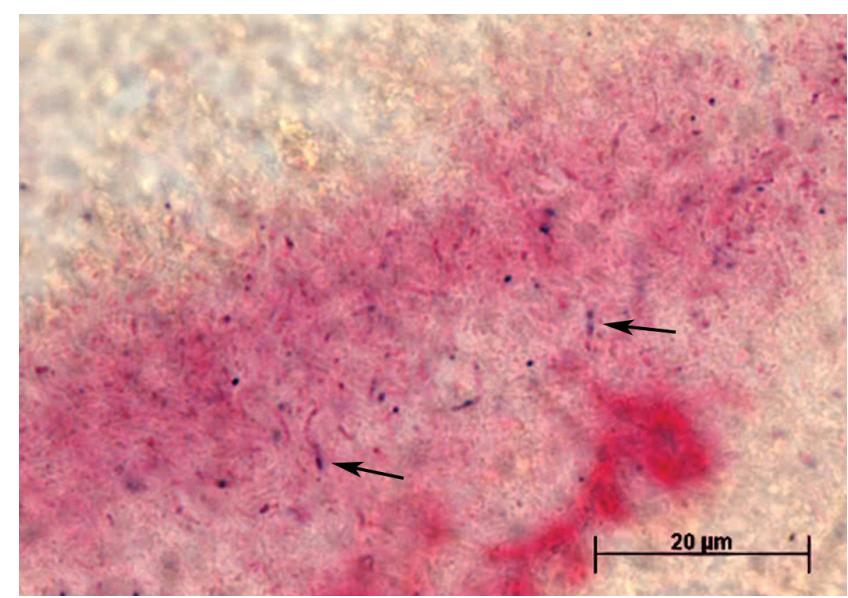

C

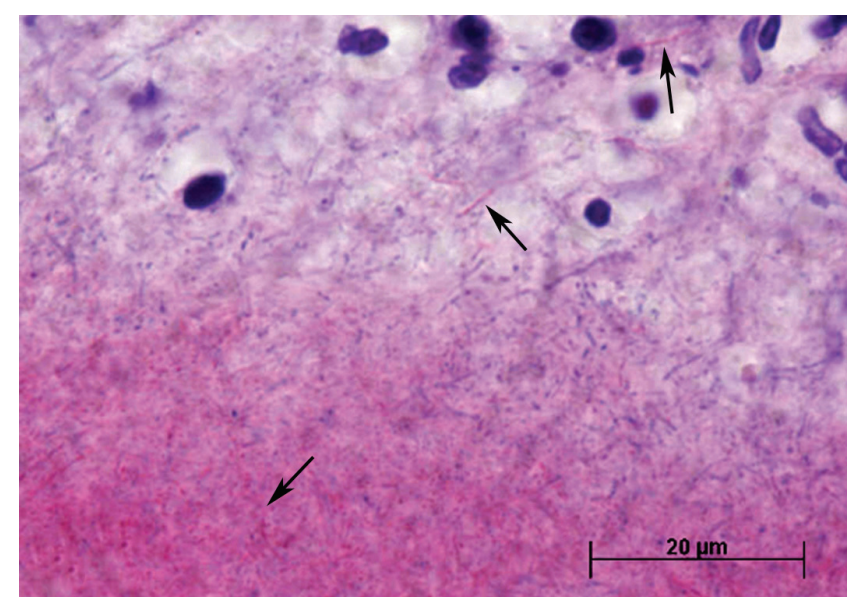

Figure 2: (A) Numerous filamentous bacteria are identified by this technique of silver impregnation. The arrows indicate the buds at right angles, characteristic of Nocardia sp. (Grocott, original magnification x100oil). (B) Presence of filamentous bacteria partially Gram-positive (arrows) (Brown-Hopes, original magnification x100oil). (C) Most of the numerous filamentous bacteria are partially acid-fast-resistant, demonstrated by shining red color of the filaments, which is characteristic of Nocardia. Arrows indicate some of these (Ziehl-Neelsen, original magnification x100oil). 
replacement due to partial dehiscence of the sewing ring causing paravalvular regurgitation. About a month later he was discharged from hospital in good general condition without fever.

\section{DISCUSSION}

There are few reports of IE due to Nocardia sp. In the literature there are two recent reviews ${ }^{4,5}$ reporting nineteen cases of nocardial endocarditis from 1970 to 2009 . To the best of our knowledge, until now no other additional case has been reported in the literature. Including the present patient, 10 cases involved natural valves and 10 other cases involved prosthetic valves, with an overall lethality of $35 \%(7 / 20)$. Eight out of thirteen survivors were surgically treated and different antibiotics were administered. The most common regimen was the association of imipenem and amikacin, followed by prolonged use of trimethoprim-sulfamethoxazole.

In general, nocardial infections affect mostly patients with immunosuppressive conditions (HIV and organ transplants), ${ }^{3}$ as well as with prolonged corticotherapy. ${ }^{6}$ Interestingly, only six out of twenty cases of nocardial endocarditis were clearly immunocompromised. While most of nocardial endocarditis patients had prior valvular heart disease (12/20), none of six immunosuppressed had cardiac predisposition for endocarditis.

Corynebacterium sp. is recognized as an uncommon agent of IE, typically affecting the left heart valves of adult men, one-third of whom have previous valvular disease. ${ }^{7}$ Rhodococcus equi organisms are coccobacilli that can be arranged at right angles to each other, leading to confusion with diphtheroid bacteria. Acid-fast-resistance and variable production of aerial hyphae can, in certain way, lead to confusion with Nocardia. In patients with cavitated pneumonia, the finding of diphtheroid bacteria in sputum and other normal commensals can raise suspicion of $R$. equi as the causal agent. The differential diagnosis of $R$. equi includes Mycobacterium tuberculosis, other non-tuberculous mycobacteria, Nocardia, fungi and diphtheroid bacteria. Because of the nature of variable acid-fast resistance and similarity to M. tuberculosis, patients initially treated as tuberculosis have been reported.
The diagnosis is established by appropriate Nocardia culture from the infection site. There is still no molecular or serological technique available for routine use. Because Nocardia grows more slowly than common bacteria, microbiology laboratories should always be notified when there is clinical suspicion of nocardiosis. An alert in the case discussed here would have been that several samples of positive blood cultures reported as diphtheroid bacteria in an immunocompromised patient should have called the attention to the ultimate identification of the agent. The morphological features described here in histological sections can be highly suggestive of Nocardia and thus guide the specific diagnosis. It should be pointed out that surgically removed specimens from endocarditis examined histologically can provide a quick indication of the infectious agent, in this case Nocardia, thus guiding therapeutics and also the appropriate culture time (at least 1 month to discard) to enable a correct diagnosis of nocardiosis. We draw attention to the fact that the histological indication of the agent was essential for adequate and timely treatment.

\section{REFERENCES}

1. Schwartz MA, Tabet SR, Collier AC et al. Central venous catheter-related bacteremia due to Tsukamurella species in the immunocompromised host: a case series and review of the literature. Clin Infect Dis 2002; 35(7):e72-7.

2. Clinical Microbiology Procedures Handbook, $2^{\text {nd }}$ Edition, Editor in Chief: Henry D. Isenberg, 2004, ASM Press

3. Lazo Torres AM, Galvez Contreras C, Collado Romacho A et al. Nocardia endocarditis in a native mitral valve. Rev Esp Cardiol 2004; 57(8):787-8.

4. Timóteo AT, Branco LM, Pinto M, Bico P et al. Nocardial endocarditis after mitral valve replacement: case report and review of the literature. Rev Port Cardiol 2010; 29(2):291-7.

5. Cargill JS, Boyd GJ, Weightman NC. Nocardia cyriacigeorgica: a case of endocarditis with disseminated soft-tissue infection. J Med Microbiol 2010; 59(2):224-30.

6. Chedid MB, Chedid MF, Porto NS et al. Nocardial infections: report of 22 cases. Rev Inst Med Trop São Paulo 2007; 49(4):239-46.

7. Belmares J, Detterline S, Pak JB et al. Corynebacterium endocarditis species-specific risk factors and outcomes. BMC Infect Dis $2007 ; 6,7: 4$ 